[Rapid Report】

\title{
Improvement of Photorefractivity in PVK Based Composite by Doping of Graphene
}

\author{
Hibiki Chiba, Kanon Hosoyama, Rika Ogawara, Shinji Kanehashi, and Kenji Ogino* \\ Graduate School of Bio-Applications and Systems Engineering, Tokyo University of Agriculture and Technology, \\ 2-24-16 Nakacho, Koganei-shi, Tokyo 184-8588 Japan
}

\begin{abstract}
The effect of graphene was investigated on photorefractivity in the composite consisting of hole transporting poly( $N$-vinyl carbazole) (PVK) (44.5 wt\%), electro-optically active 4-(azepan-1-yl) benzylidenemalononitrile (7-DCST) (35.0 wt\%), plasticizing dibutyl phthalate (DBP) (20.0 wt\%), and photosensitizing fullerene $\left(\mathrm{C}_{60}\right)(0.5 \mathrm{wt} \%)$ by changing the amount of graphene from 0 to 0.1 wt\% and monitoring the two beam coupling gain coefficient. It is found that the gain coefficient drastically increased by the addition of graphene and reached maximum at the content of around 0.005-0.007 wt\% (e.g., it was $112 \mathrm{~cm}^{-1}$ at $41.7 \mathrm{~V} / \mu \mathrm{m}$ at $0.005 \mathrm{wt} \%)$. Beyond the maximum, the gain coefficient went through the local minimum value at around $0.05 \mathrm{wt} \%$, and then gradually increased till $0.1 \mathrm{wt} \%$. These results suggested that at the lower content, graphene worked as trap sites, and at the higher content, the nature of trap sites was changed by the onset of the overlap of graphene sheets.
\end{abstract}

(Received 29 July, 2018; Accepted 16 August, 2018)

\section{Introduction}

Photorefractivety has been originally observed in inorganic crystals possessing both a photoconductivity and a linear electro-optical (EO) response [1]. Illumination of a photorefractive (PR) material by a spatially modulated light generates the free carriers in the bright part region, and one of the resulting carriers (e.g. hole) drifts and/or diffuses, and is trapped to form the modulated space charge field. Due to the EO activity, the refractive index is modulated in the material. The resulting index modulation brings about an optical diffraction, and an optical photoamplification as a result of asymmetric energy transfer, which means that one of two beams (pumping beam) forming a spatially modulated interference pattern transfers its energy to another beam. This asymmetric energy transfer is a unique characteristic of PR effect, and is originated from the phase shift between the interference pattern and the resulting refractive index modulation. The photoamplification is experimentally evaluated with two beam coupling $(2 \mathrm{BC})$ gain representing the extent of asymmetric energy transfer. Since the first report for photorefractive (PR effect) in an organic material [2], a variety of organic PR materials have been intensively investigated [3]. Among them, composites consisting of a hole transporting polymer doped with an EO active chromophore, appropriate amount of a plastisizer, and small amount of a photosensitizing electron acceptor have exhibited higher performance such as higher diffraction efficiencies, larger 2BC gains, and shorted response time [4-9]. For example, the hole transporting poly( $N$-vinyl carbazole) (PVK) based composite containing an EO active chromophore, a plasticizer, and a small amount of electron acceptor as a photosensitizer exhibited a diffraction efficiency approaching $100 \%$ and a $2 B C$ gain of more than $200 \mathrm{~cm}^{-1}$ [4]. As an electron acceptor, $\mathrm{C}_{60}$ has been most commonly utilized in high perfomance materials. More recently the utilization of chemical analogues such as more soluble [6,6]-phenyl $\mathrm{C}_{61}$-butyric acid methyl ester (PCBM) has been reported [8, 9]. Carbon nanotubes (CNTs) in the same family of carbon allotropes have improved the PR performance from the viewpoint of photosensitizers [10].

Graphene also belongs to the family of

\# corresponding author: Kenji Ogino (E-mail: kogino@cc.tuat.ac.jp; Tel: +81-42-388-7404; Fax: +81-42-388-7404) 
nanocarbon materials, and consists of all sixmembered rings with $\mathrm{sp}^{2}$ carbons having a two dimensional sheet structure. Since the first isolation of a thin flake graphene in 2004 [11], it has been proved that graphene posesses the remarkable characteristics of electronic, mechanical, and chemical properties. Individual graphene sheet exhibits high thermal conductivity [12], high Young modulus [13], and high electron mobility [14]. Graphene has been applied to PR materials as an additive. Grishina et al. reported the photorefractivity for the composites based on conventional PVK / graphene [15, 16]. Since these composites contained no EO chromophore, the mechanism of the formation of index grating was unclear. More recently Chantharasupawong et al. investigaed PR performances of a graphene doped poly(acrylic tetraphenyldiaminobiphenyl) (PATPD) composites with EO chromophore [4-(azepan-1-yl) benzylidene]malononitrile, 7-DCST), and reported the improved response time due to the enhancement of photocarrier generation [17].

In this communication, we investigated the effect of graphene content on 2BC gain coefficient for the conventional PR composites, PVK (44.5 wt\%) / 7-DCST) (35.0 wt\%), dibutyl phthalate (DBP) (20.0 wt\%), and fullerene $\left(\mathrm{C}_{60}\right)(0.5 \mathrm{wt} \%)$. It was found that the addition of graphene enhanced $2 \mathrm{BC}$ gain exhibiting the strong content dependency.

\section{Experimental Section}

The chemical structures of compounds used here are shown in Fig. 1(a). Commercially available poly ( $N$-vinyl carbazole) (PVK) (Mw: 1,100,000) (SigmaAldrich), dibutyl phthalate (DBP) (TCI), 1,1,2,2tetachloroethane (TCI), fullerene $\left(\mathrm{C}_{60}\right)$ (Kanto Chemical) and graphene nanopowder (EMJapan) were used as received. According to the catalogue for graphene, the average flake thickness and the average lateral size are $1.6 \mathrm{~nm}$ (less than 3 layers), and $10 \mu \mathrm{m}$, respectively. The specific surface area is 510 m²/g. 4-(Azepan-1-yl)benzylidenemalononitrile (7-DCST) was synthesized as described in the literature [18]. The 1,1,2,2-tetrachloroethane solution of $\mathrm{C}_{60}(1 \mathrm{mg} / \mathrm{mL})$ and the dispersion of graphene $(0.4 \mathrm{mg} / \mathrm{mL})$ were prepared with the aid of ultrasonic treatment.

PR composites and devices were fabricated as follows. PVK (0.89 g), 7-DCST (0.70 g), DBP (0.40 g) was dissolved in $10 \mathrm{~mL}$ of $\mathrm{C}_{60}$ solution, and then the (a)
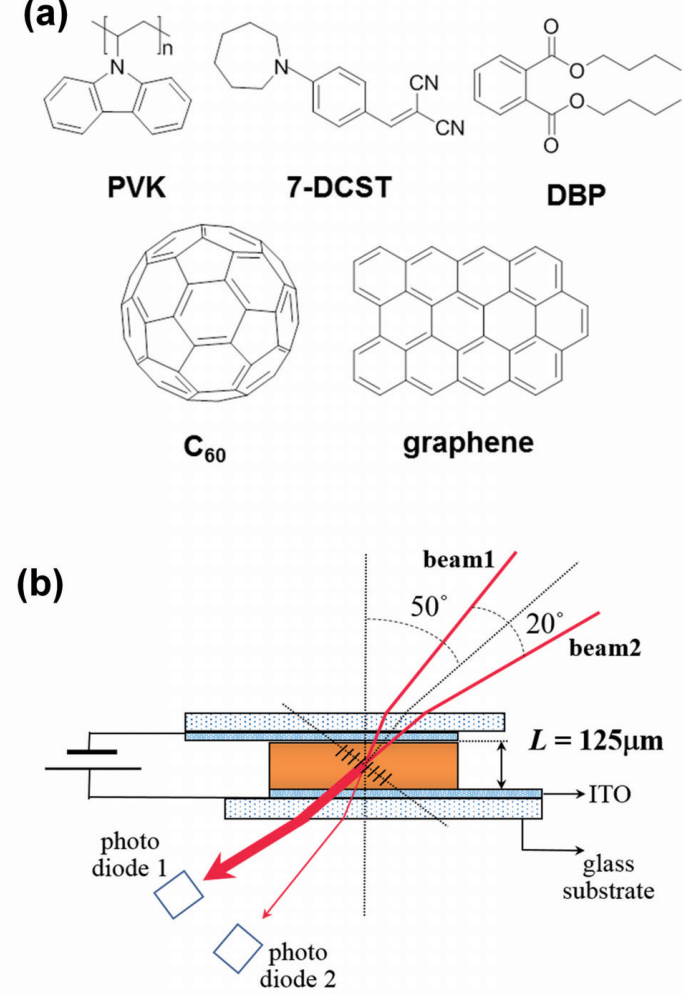

Fig. 1 Chemical structures of compounds (a) and schematic representation of experimental setup for two beam coupling measurement (b) in this study.

predetermined amount of graphene dispersion $(0-500 \mu \mathrm{L})$ was added to the solution with a microsyringe. After the concentration to around the one-third volume, the small portion of the solution was placed on an indium tin oxide (ITO, $30 \Omega$ /sq.) covered glass. After the evaporation of most of the solvent on a hot plate at $60^{\circ} \mathrm{C}$, the sample was dried with the vacuum oven at $50^{\circ} \mathrm{C}$ for $2 \mathrm{~h}$. Sandwiched type of sample was fabricated with two glasses with deposited with the composites with the hot plate at $60^{\circ} \mathrm{C}$ The sample thickness was adjusted to approximately $125 \mu \mathrm{m}$ a Kapton ${ }^{\circledR}$ spacer. To verify the reproducibility, at least three devices were fabricated at the same composition.

Experimental setup for the two-beam coupling (2BC) measurement using mutually coherent beams $(633 \mathrm{~nm})$ from an NEC GLS-5410 He-Ne laser was almost the same as described elsewhere [19]. The schematic representation of the setup is shown in Fig. 1 (b). All the $2 \mathrm{BC}$ experiments were performed at room temperature $\left(22 \pm 2^{\circ} \mathrm{C}\right)$. The intensities of two writing beams (p-polarized) were adjusted to the same values $\left(110 \mathrm{~mW} / \mathrm{cm}^{2}\right)$. The angle between two writing beams outside the sample was $20^{\circ}$ and the tilt angle was $50^{\circ}$. Photodiodes (Hamamatsu Photonics, 
S2281) were utilized to monitor the transmitted beam intensities. Absorption coefficients at $633 \mathrm{~nm}$ and glass transition temperature were determined using a JASCO V-570 UV/VIS/NIR spectrophotometer, and a Rigaku Thermo Plus DSC8230, respectively.

\section{Results and discussions}

\subsection{Absorption and glass transition}

Apparently homogeneous dispersion of graphene was obtained by the ultrasonic treatment. Since the content of graphene in the composite was low (at most $0.1 \mathrm{wt} \%)$, the optical quality of composite films was obtained in the concentration range of graphene we examined. Fig. 2 shows the absorption profiles without and with graphene (0.007 wt\%) (a), and the dependence of graphene content on the absorbance
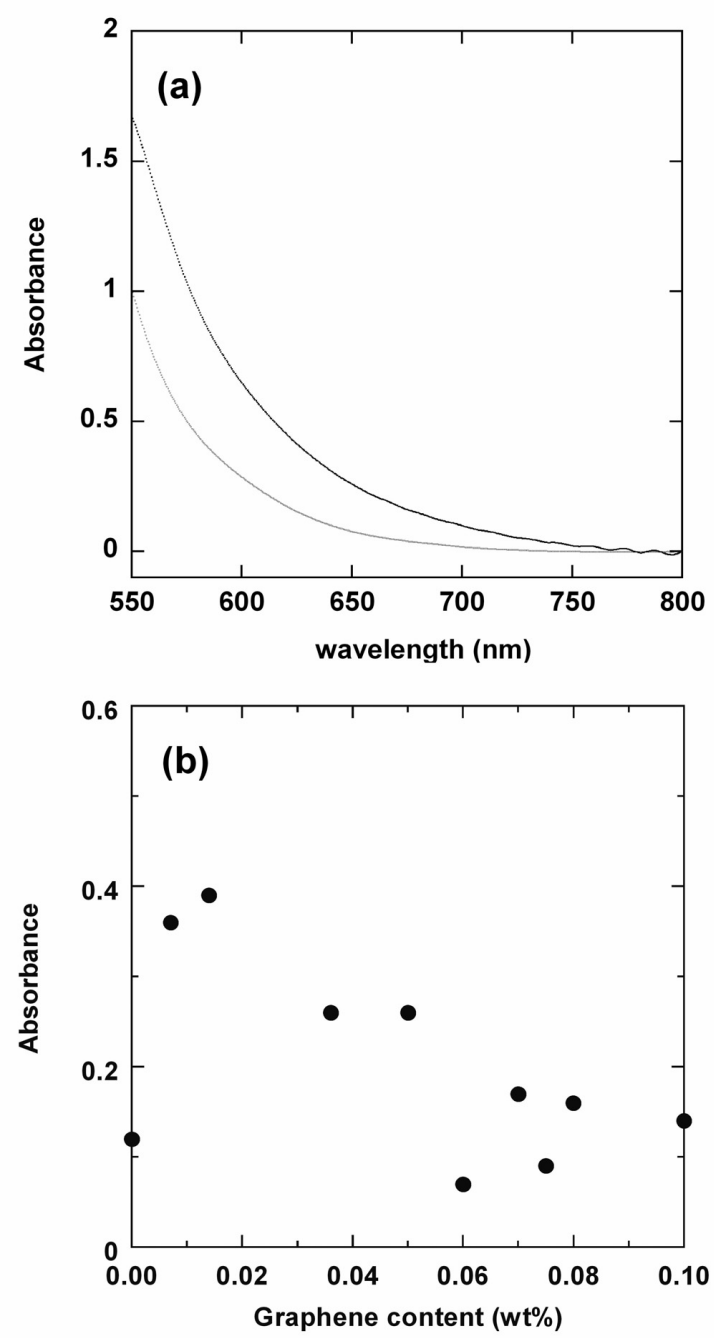

Fig. 2 Absorption profiles of composites without (blue) and with graphene (0.007 wt\%) (black) (a) and relationship between graphene content and absorbance at $633 \mathrm{~nm}$ (b) for PR devices. The film thickness; $125 \mu \mathrm{m}$. Measurements were conducted with two ITO covered glasses as a reference. for PR devices at $633 \mathrm{~nm}$ (b). The graphene doped composite films exhibited a stronger absorption tail in the visible region compared with the composite without graphene as shown in Fig. 2 (a). As shown in Fig. 2 (b), no Lambert-Beer relationship was obtained, i.e., there seems the trend that absorbance got smaller as the graphene content increased. Furthermore, the absorption coefficients were larger compared with the composites consisting of poly(dimethylsiloxane) (PDMS) and graphene [20], where no specific interaction exists between both components. Graphene/PDMS composite film with $100 \mu \mathrm{m}$ in thickness exhibited absorbance increasing from 0.016 to 0.088 with increasing graphene loading from 0.02 to $0.1 \mathrm{wt} \%$. Although the exact value of the coefficient of graphene/polymer composite may depend on the flake thickness and size distribution [21], and the contribution of fullerene should be taken into consideration, the absorbance in our composite enhanced by a factor of 10 at $0.02 \mathrm{wt} \%$ compared with the PDMS/graphene composite even after the contribution of fullerene was eliminated. These results suggest that charge transfer interaction between PVK or 7-DCST and graphene exists especially in the low content of graphene as suggested in the literature [17]. Further investigation is necessary to interpret the enhanced absorbance in this system. Glass transition temperatures of composites were in the range of $-35 \sim-25^{\circ} \mathrm{C}$, and no significant dependence of the graphene content was observed.

\subsection{Two beams coupling gain}

Fig. 3 shows the intensity profiles of two transmitted beams in $2 \mathrm{BC}$ experiments for the

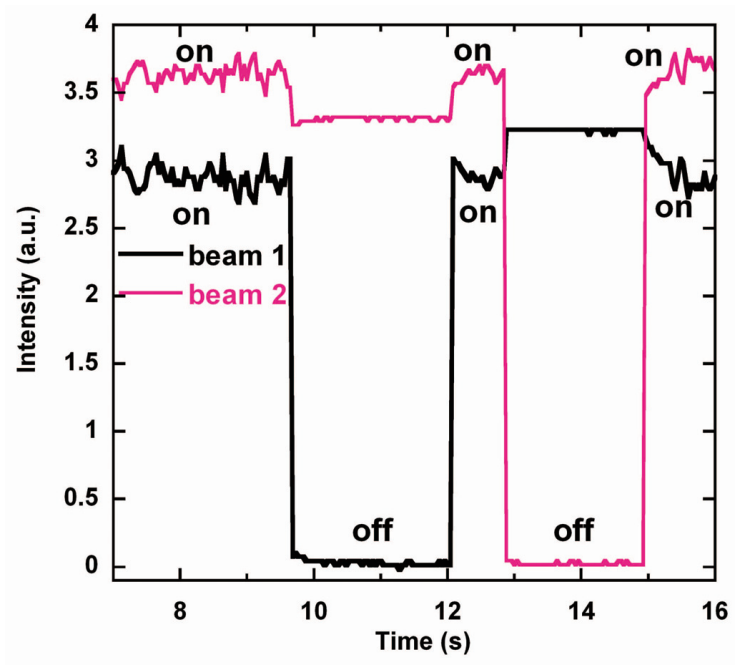

Fig. 3 Asymmetric energy transfer observed in the composite containing $0.005 \mathrm{wt} \%$ of graphene at $33.3 \mathrm{~V} / \mu \mathrm{m}$ 
composite with $0.005 \mathrm{wt} \%$ of graphene. When beam 1 was off at $9.6 \mathrm{~s}$ for the pre-illuminated device, the intensity of beam 2 decreased. On the other hand, when beam 2 was off at $12.8 \mathrm{~s}$, the intensity of beam 1 increased. Asymmetric energy transfer was observed, i.e., beam 1 lost the energy, and beam 2 gained the energy. When the intensities of two writing beams were equal, the coupling gain coefficients were determined with Equation (1),

$$
\Gamma=(\cos \theta / L)\left[\ln \left(\gamma_{0}\right)-\ln \left(2-\gamma_{0}\right)\right]
$$

where $L$ is the sample thickness, $\gamma_{0}=I / I_{0}$ is the beam coupling ratio where $I_{0}$ is the intensity without the pump beam, and $I$ is the intensity with pump beam on, and $\theta$ is the incident angle of beam inside the composite film. The electric field dependences of gain coefficients are shown in Fig. 4 observed in the
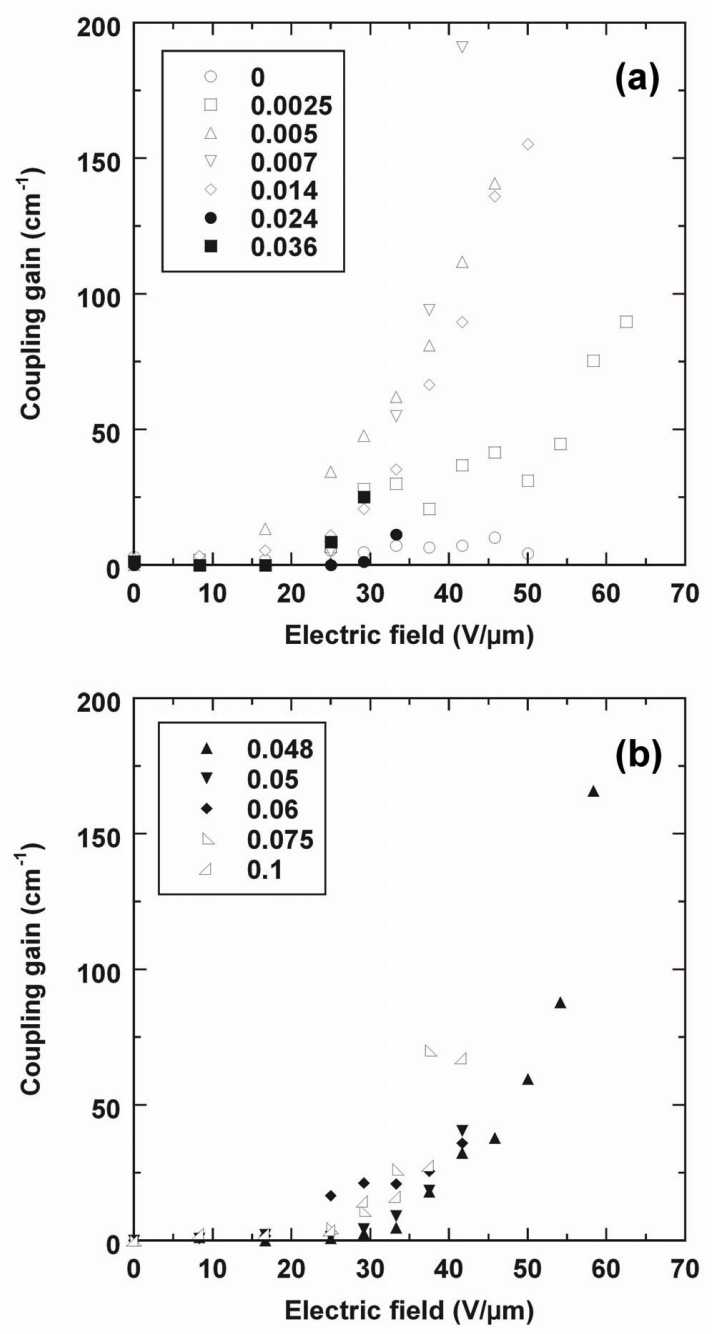

Fig. 4 Electric field dependence of coupling gain coefficient for PR devices with low (a) and high (b) graphene content. The numbers in the legend represent the concentration of graphene (wt\%). composites with lower graphene content (a), and higher one (b).

It is notworthy that the composites doped with graphene exhibited the larger coupling gain compared with the undoped composite. The dependence of graphene content on the coupling gain coefficients seems complicated. Although the composite with $0.05 \mathrm{wt} \%$ of graphene showed the large coupling gain coefficient in the higher electric field region (over $50 \mathrm{~V} / \mu \mathrm{m}$ ), relatively smaller coupling gain was observed in the lower electric field region.

As shown in Fig. 5, the coupling gain coefficients are plotted against the graphene contents for the different electric fields to elucidate the dependence of the graphene content. It is found that the gain coefficient drastically increased by the addition of graphene and reached maximum at the content of around $0.005-0.007 \mathrm{wt} \%$. Beyond the maximun, the gain coefficients gradually decreased and reached the minimun values around at $0.05 \mathrm{wt} \%$. Beyond this content, the gain coefficients seemed to increase with the graphene content. It is considered that at the lower graphene content, graphene played a role of trap sites of holes. According to the Kukhtarev model [22], the magnitude of the space charge field $\left(E_{\mathrm{sc}}\right)$ established in PR materials is described in Equation (2):

$$
\left|E_{\mathrm{SC}}\right|=m\left(\frac{\left(E_{0}{ }^{2}+E_{\mathrm{D}}{ }^{2}\right)}{\left(1+E_{\mathrm{D}} / E_{\mathrm{q}}\right)^{2}+\left(E_{0} / E_{\mathrm{q}}\right)^{2}}\right)^{1 / 2}
$$

where $m$ is the fringe visibility, $E_{0}$ is the applied electric field, $E_{\mathrm{D}}$ is the diffusion field, and $E_{\mathrm{q}}$ is the

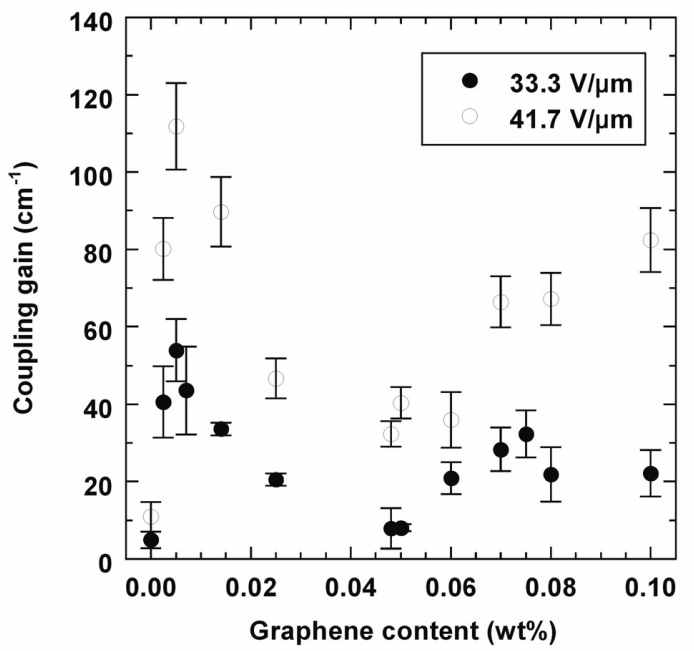

Fig. 5 Relationship between coupling gain coefficient and graphene content. White and black circles represent the results at 33.3 and $41.7 \mathrm{~V} / \mu \mathrm{m}$, respectively. 
limiting space charge field. The diffusion field is afforded by

$$
E_{\mathrm{D}}=\frac{2 \pi k_{\mathrm{B}} T}{\Lambda e}
$$

where $\Lambda$ is the grating spacing of the original interference pattern, $e$ is the electronic charge, $k_{\mathrm{B}}$ is Boltzmann constant, and $T$ is the absolute temperature. The limiting space charge field is afforded by

$$
E_{\mathrm{q}}=\frac{2 \Lambda e N_{e f f}}{\varepsilon}
$$

where $N_{\text {eff }}$ is the effective trap density, and $\varepsilon$ is the dielectric constant. The magnitude of the coupling gain is proportional to the product of $E_{\mathrm{SC}}$ and $\sin \varphi$, where $\varphi$ is the phase shift between the interference pattern and the resulting sinusoidal $E_{\mathrm{SC}}$ pattern [22, 23]. The phase shift $\varphi$ :

$$
\varphi=\arctan \left[\frac{E_{\mathrm{D}}}{E_{0}}\left(1+\frac{E_{\mathrm{D}}}{E_{\mathrm{q}}}+\frac{E_{0}{ }^{2}}{E_{\mathrm{D}} E_{\mathrm{q}}}\right)\right]
$$

The space charge field, $E_{\mathrm{SC}}$ increases and $\varphi$ decreases with the increase of $E_{\mathrm{q}}$. It is predicted that the maximum value for the coupling gain is observed when the trap density changes. The experimental results presented here can be quanlitatively explained by the assumption that the the doped graphene worked as trap sites for hole transporting. Monolayer graphene exhibits no electronic band gap, but a band gap in graphene can emerge as a result of the interaction with some substances or the adsorption of organic molecule [24]. Therefore, it is reasonable that graphene in the composite possessed the certain band gap, and the ability of the hole trap.

The origin of the experimental results that gain coefficients increased from $0.05 \mathrm{wt} \%$ to $0.1 \mathrm{wt} \%$ is still unclear. From the absorption data, it is suggested that the band structure related to graphene was different from that of lower content. Furthermore, it is reported that the sharp increase of the electric conductivity was observed around 0.1 vol\% for the poly(vinyl chloride) / graphene composite film, and the percolation threshhold was around $0.6 \mathrm{vol} \%$ [25]. This suggests that the significant overlap of graphene sheets started in the concentration range of 0.05-0.1 wt\% in this study. It is considered that the onset of the overlap altered the band structure leading to the change of the nature of trap sites.

\section{Conclusions}

The doping of graphene to conventional PVK based composites improved PR performance. The relationship between the content of graphene and the coupling gain suggests that doped graphene worked as the trap sites resulting in the enhancement of the gain coefficients. The dependence of the graphene content on the absorbance indicated that the band structure altered probably due to the partialy overlapped and aggregated structure of graphene in the concentration range of $0.05-0.1 \mathrm{wt} \%$. It is suggested that the resulting overlap also changed the nature of trap sites leading to the gradual increase of the gain coefficient.

\section{References}

1. P. Yeh, "Introduction to Photorefractive Nonlinear Optics”, Wiley, New York (1993).

2. K. Sutter, J. C. Scott, R. Günter, Solid State Commun., 74, 867 (1990).

3. N. Tsutsumi, Polym. J., 42, 572 (2016).

4. K. Meerholz, B. L. Volodin, Sandalphon, B. Kippelen, N. Peyghambarian, Nature, 371, 497 (1994).

5. K. Ogino, T. Nomura, T. Shichi, S.-H. Park, H. Sato, T. Aoyama, T. Wada, Chem. Mater., 9, 2768 (1997).

6. H. Chun, I. K. Moon, D.-H. Shin, and N. Kim, Chem. Mater., 13, 2813 (2001).

7. Z. Cao, Y. Abe, T. Nagahama, K. Tsuchiya, and K. Ogino, Polymer, 54, 269 (2013).

8. N. Tsutsumi, K. Kinashi, K. Masumura, K. Kono, Opt. Express, 23, 25158 (2015).

9. P.-A. Blanche, B. Lynn, D. Churin, K. Kieu, R. A. Norwood, N. Peyghambarian, Sci. Rep., 6, 29027 (2016).

10. N. K. Lingam, S. Kalghatgi, J. G. Winiarz, J. Appl. Phys., 109, 023106 (2011).

11. K. S. Novoselov, A. K. Geim, S. V. Morozov, D. Jiang, Y. Zhang, S. V. Dubonos, I. V. Grigorieva, A. A. Firsov, Science, 306, 666 (2004).

12. A. A. Balandin, S. Ghosh, W. Bao, I. Calizo, D. Teweldebrhan, F. Miao, C. N. Lau, Nano Letters, 8, 902 (2008).

13. C. Lee, X. D. Wei, J. W. Kysar, J. Hone, Science, 321, 385 (2008).

14. K. I. Bolotin, K. J. Sikes, Z. Jiang, M. Klima, G. Fudenberg, J. Hone, P. Kim, H. L. Stormer, Solid State Commun., 146, 351 (2008). 
15. A. D. Grishina, T. V. Krivenko, V. V. Savel'ev, R. W. Rychwalski, A. V. Vannikov, High Energy Chem., 47, 46 (2013).

16. A. D. Grishina, T. V. Krivenko, V. V. Savel'ev, R. W. Rychwalski, A. V. Vannikov, High Energy Chem., 47, 187 (2013).

17. P. Chantharasupawong, C. W. Christenson, R. Philip, L. Zhai, J. Winiarz, M. Yamamoto, L. Tetard, Rahul R. Nair, J. Thomas, J. Mater. Chem. C, 2, 7639 (2014).

18. H. N. Giang, K. Kinashi, W. Sakai, N. Tsutsumi, Polym. J., 46, 59 (2014).

19. K. Okamoto, T. Nomura, K. Ogino, H. Sato, Chem. Mater., 11, 3279 (1999).
20. K. Zeranska-Chudek, A. Lapinska, A. Wroblewska, J. Judek, A. Duzynska, M. Pawlowski, A. M. Witowski, M. Zdrojek, Sci. Rep., 8, 9132 (2018).

21. R. Su, S. F. Lin, D. Q. Chen, G. H. Chen, J. Phys. Chem. C, 118, 12520 (2014).

22. N. V. Kukhtarev, V. B. Markov, S. G. Odulov, M. S. Soskin, V. L. Vinetskii, Ferroelectrics, 22, 949 (1979).

23. W. E. Moerner, A. Grunnet-Jepsen, C. L. Thompson, M. S. Bratcher, R. J. Twieg, Proc. SPIE, 3147, 84 (1997).

24. L. Kong, A. Enders, T. S. Rahman, P. A. Dowben, J. Phys.: Condens. Matter., 26, 443001 (2014).

25. S. Vadukumpully, J. Paul, N. Mahanta, S. Valiyaveettil, Carbon, 49, 198 (2011). 